\title{
Paradoxical reaction in the form of pleural effusion after onset of anti-tuberculous medication for tubercular lymphadenitis
}

\author{
Authors: Ambarish Bhattacharya ${ }^{A}$ and Suranjan Mukherjee ${ }^{B}$
}

\begin{abstract}
We present the case of a 26-year-old Indian male who developed pleural effusion while undergoing treatment for tuberculous lymphadenitis. We describe the work-up for his condition and how he was managed. The possibility of development of a paradoxical reaction in the form of pleural effusion after initiation of anti-tuberculous therapy has to be kept in mind while treating such patients.
\end{abstract}

KEYWORDS: Paradoxical reaction, pleural effusion, tubercular lymphadenitis, tuberculosis

\section{Case presentation}

A 26-year-old Indian male presented with gradually increasing painless swelling behind the right jaw, associated with a daily low-grade fever in the evening. There was no history of any other swelling elsewhere. There was no history of cough, breathlessness, night sweats or weight loss. He did not have any complaints relating to his throat, gums, teeth, ear or scalp. He was a non-smoker with no history of illicit drug or alcohol intake. There was no history of contact with tuberculosis.

Clinical examination revealed an enlarged non-tender lymph node $(1 \mathrm{~cm} \times 2.5 \mathrm{~cm})$ in the right superior deep cervical lymph node region (Fig 1) with no other palpable lymph node. He was afebrile; his pulse was $70 \mathrm{bpm}$ and blood pressure was 110/80 mmHg. Systemic examination did not reveal any abnormality other than mild pallor.

\section{Differential diagnosis}

The most likely diagnosis was tuberculous lymphadenitis given the prevalence of this disease in this subcontinent. However, a thorough examination of the patient to look for infective and neoplastic lesions in the scalp, oral cavity and throat is necessary, along with examination for enlarged lymph nodes and hepatosplenomegaly.

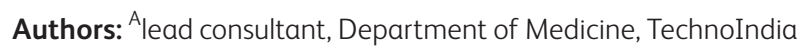
DAMA Hospital and Medical research center, Kolkata, India; ${ }^{\text {s }}$ senior consultant, Department of Respiratory Medicine, AMRI Hospitals Dhakuria, Kolkata, India

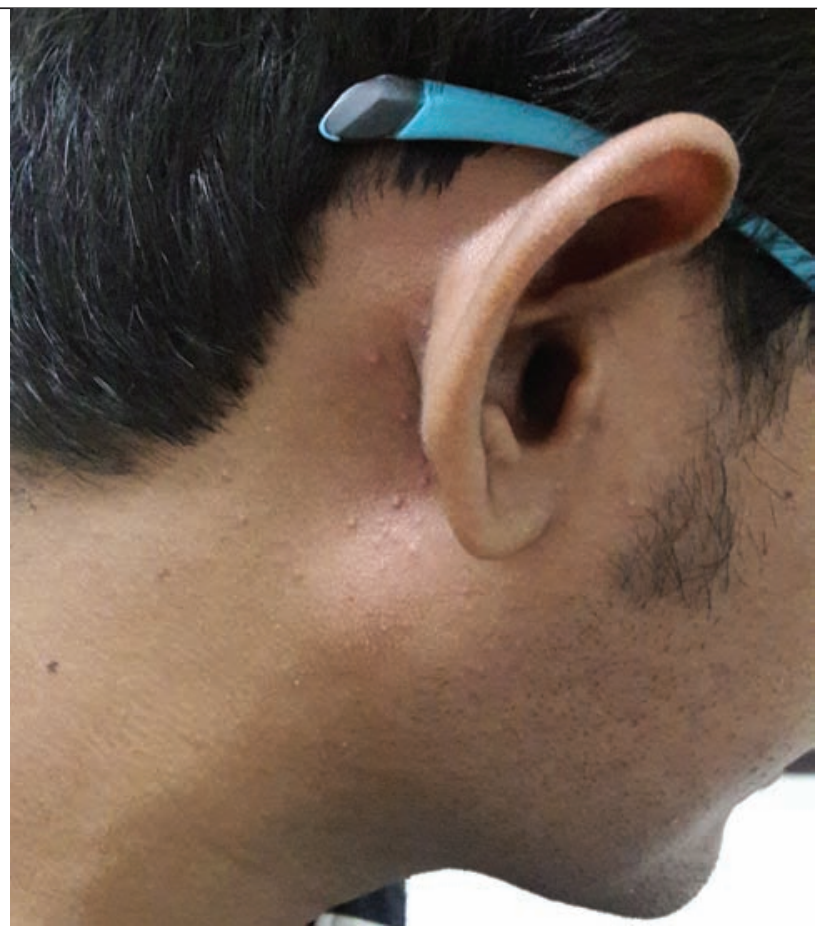

Fig 1. Patient on initial presentation.

\section{Initial management and prognosis}

Fine needle aspiration cytology from the lymph node, complete haemogram, HIV 1 and 2, liver function test, serum creatinine, serum glucose, chest radiograph and ultrasonogram of the whole abdomen were performed.

Haemoglobin was $8.3 \mathrm{~g} / \mathrm{dL}$, erythrocyte sedimentation rate was $110 \mathrm{~mm}$ in hour 1 and C-reactive protein was $60 \mathrm{mg} / \mathrm{L}$. Fine needle aspiration cytology showed caseation necrosis along with giant cells. WHO Xpert/TB/RIF assay on the sample confirmed the presence of Mycobacterium tuberculosis (rifampicin sensitive). Combination anti-tuberculous therapy (Category 1, WHO: isoniazide+rifampicin+pyrazinamide+eth ambutol (HRZE)) was started.

After 1 month he returned with progressive breathlessness, chest discomfort and cough. However, he had gained $2 \mathrm{~kg}$ in 


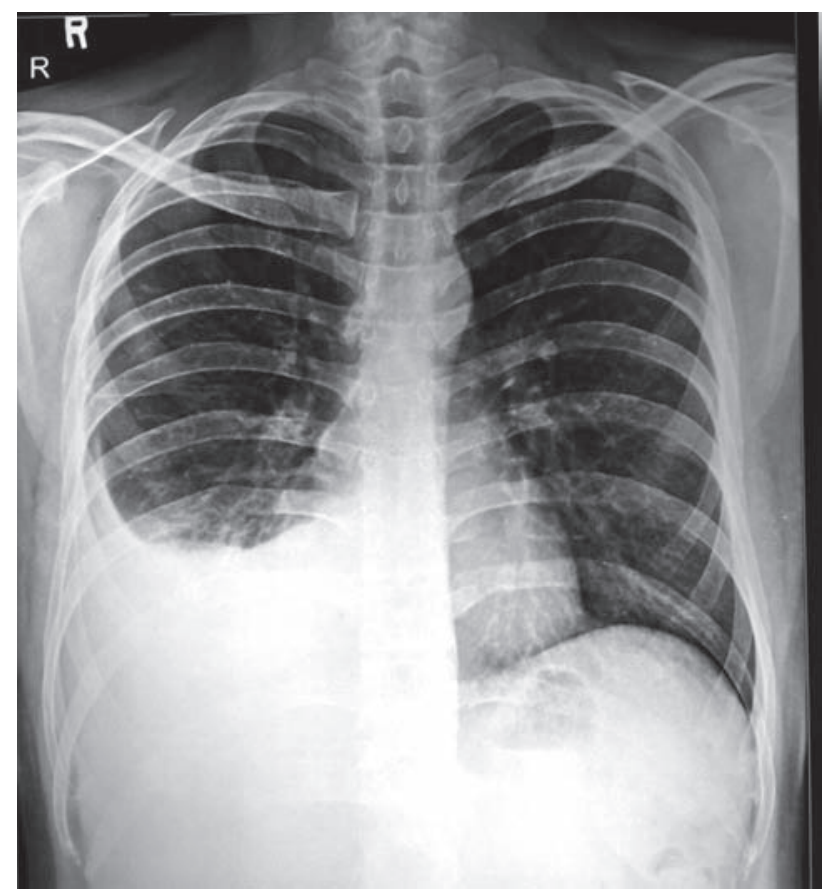

Fig 2. Chest X-ray after 1 month showing pleural effusion.

weight, was afebrile, had better appetite and the lymph node had decreased in size. Chest X-ray showed moderate sized right-sided pleural effusion (Fig 2). Ultrasonogram-guided diagnostic thoracentesis showed exudative straw coloured fluid with high lymphocyte count but low adenosine deaminase and a negative Xpert/TB assay (Tables 1 and 2).

Table 1. Parameters of examination of pleural fluid

\begin{tabular}{ll} 
Parameter & Value \\
Cell count & $2000 / \mathrm{mm}^{3}$ \\
Cell type & $\mathrm{L} 74$ \\
& $\mathrm{~N} 18$ \\
& $\mathrm{E} 7$ \\
& $\mathrm{M} 1$ \\
Sugar & $89 \mathrm{mg} / \mathrm{dL}$ \\
Protein & $6.7 \mathrm{~g} / \mathrm{dL}$ \\
LDH & $419 \mathrm{U} / \mathrm{L}$ \\
Albumin & $2 \mathrm{~g} / \mathrm{dL}$ \\
ADA & $27.2 \mathrm{U} / \mathrm{L}$ \\
Gram stain & No organism present \\
ZN stain & \\
Culture & \\
Pap stain & Shows mature lymphocytes \\
\hline ADA = adenosine deaminase; LDH = lactate dehydrogenase; ZN = Ziehl- \\
Neelsen & No malignant cells seen \\
\hline
\end{tabular}

Table 2. Blood results sent during first follow-up

\begin{tabular}{|c|c|c|}
\hline Parameter & Value & Reference \\
\hline $\mathrm{Hb} \%, \mathrm{~g} / \mathrm{dL}$ & 11.2 & $14-16$ \\
\hline TLC, $\mu \mathrm{L}$ & 7,300 & $4,000-11,000$ \\
\hline \multirow[t]{4}{*}{ DC } & N60 & \\
\hline & L34 & \\
\hline & M4 & \\
\hline & E2 & \\
\hline PLC, $\mu \mathrm{L}$ & 160,000 & $150,000-450,000$ \\
\hline $\mathrm{ESR}, \mathrm{mm}$ & 40 & $<20$ \\
\hline CRP, mg/L & 40.3 & $<5$ \\
\hline $\mathrm{RBS}, \mathrm{mg} / \mathrm{dL}$ & 120 & $<110$ \\
\hline Creatinine, mg/dL & 1.2 & $<1.5$ \\
\hline Bilirubin total, mg/dL & 1.0 & $<1.5$ \\
\hline Bilirubin direct, mg/dL & 0.5 & \\
\hline ALT, U/L & 100 & $<40$ \\
\hline ALP, IU/L & 74 & $40-120$ \\
\hline Total Protein, g/dL & 7.89 & $6-8$ \\
\hline Albumin, g/dL & 3.44 & $3.5-5$ \\
\hline LDH, IU/L & 222 & $<250$ \\
\hline
\end{tabular}

$\mathrm{ALP}=$ alkaline phosphatase; $\mathrm{ALT}=$ alanine transaminase; $\mathrm{CRP}=\mathrm{C}$-reactive protein; $D C=$ differential count of white blood cell count; $E S R=$ erythrocyte sedimentation rate; $\mathrm{Hb} \%$ = haemoglobin; $\mathrm{PLC}=$ platelet count; $\mathrm{RBS}=$ random blood sugar; $T L C=$ total leukocyte count

\section{Case progression and outcome}

A diagnosis of paradoxical reaction or IRIS (immune reconstitution inflammatory syndrome) was made and the patient was started on prednisolone $(0.75 \mathrm{mg} / \mathrm{kg} /$ day $)$. The combination HRZE was also continued. Therapeutic thoracentesis was not needed. Within 2 weeks, symptoms improved significantly and chest X-ray showed complete resolution of effusion. HRZE was continued and prednisolone gradually tapered off by $5 \mathrm{mg} /$ week. Currently, the patient is asymptomatic and has been switched to maintenance phase.

\section{Discussion}

IRIS was the term originally used to describe the inflammatory pattern, which follows initiation of highly active antiretroviral therapy (HAART) in patients infected with HIV. It is paradoxical in the sense that there is worsening of clinical features in spite of appropriate therapy. ${ }^{1}$

A similar paradoxical inflammatory response, with an incidence of up to $20 \%$, has also been reported following initiation of anti-tuberculous medication in patients infected with $M$ tuberculosis. ${ }^{2}$ Researchers believe this to be an exacerbation of the immune response to the $M$ tuberculosis antigen when effective anti-tuberculous treatment is initiated. ${ }^{3}$ Constitutional symptoms like fever, malaise and weight loss can develop along with worsening of existing radiographic abnormalities, which can progress to severe respiratory distress and adult respiratory distress syndrome in rare cases. 
Often, the clinical pattern is dictated by the original site of the $M$ tuberculosis infection, but it may affect sites not originally affected by the infection; for example, in extra-pulmonary tuberculosis, the clinical pattern may include worsening lymphadenitis (computerised tomography scan may show new necrosis in lymph nodes), worsening pleural effusion (or appearance of new effusion on the contralateral side) and expansion of previous intracranial tuberculomas (presenting with new onset/worsening headaches). The respiratory and central nervous system are the most commonly reported sites for development of paradoxical reaction; other sites that could be affected are the lymph nodes, skin, soft tissue, bone, tendons and abdomen. ${ }^{4}$

Paradoxical reaction following anti-tuberculous treatment is common in both HIV and non-HIV infected individuals. Patients with disseminated tuberculosis, extra-pulmonary tuberculosis or tuberculosis co-infected with HIV are more likely to develop paradoxical reactions. Risk factors for paradoxical reactions in patients without HIV include baseline anaemia, hypoalbuminaemia and lymphopenia. ${ }^{5}$ The mechanism for paradoxical reaction after starting tuberculosis treatment is not well understood but it is very likely immune mediated. At the time of paradoxical deterioration, a concomitant increase in the lymphocyte count and conversion of the tuberculin skin test is observed. ${ }^{4}$ Initiation of anti-tuberculous therapy and subsequent reduction in mycobacterial load leads to reversal of the immune response. The clinical severity of a paradoxical reaction is probably determined by the magnitude and timing of the immune response; an overwhelming response may produce excessive immunopathological damage at the tissue level. The median time to development of paradoxical reaction is 60 days (range 14-270 days) in HIV negative individuals. ${ }^{4}$

Differential diagnosis of a paradoxical reaction would include secondary infections, adverse drug reactions, drug resistance and poor compliance. Diagnosis of paradoxical reaction is by exclusion; therefore, investigations should be directed to exclude the above conditions. Gram staining, acid fast staining, as well as bacterial and mycobacterial cultures obtained from the involved organs in these cases are mostly negative; however, occasionally acid-fast bacilli might be seen. Although even then, there would be no evidence of resistance to the drugs used in that particular regimen on drug sensitivity testing.

Most of the non-severe paradoxical reaction does not require any specific treatment once the diagnosis is established. Patients need reassurance and anti-tuberculosis therapy needs to be continued. A short course of oral corticosteroids, which is tapered off, can be considered. Severe clinical deterioration is rare but has been reported; this can include obstructive hydrocephalus secondary to enlargement of tuberculomas, massive pleural effusion and development of deep-seated abscesses. In addition to systemic steroids, these patients might need surgical drainage procedures. Most patients will recover uneventfully with either conservative treatment or a combination of medical and surgical management. Only a few cases with central nervous system involvement result in residual neurological deficits. ${ }^{6}$

\section{Key learning points}

> Paradoxical reaction can develop in patients after starting anti-tuberculous therapy similar to HAART therapy in patients with HIV.

> Paradoxical reaction includes enlargement of the original lesion (increase in size of lymph nodes) or development of new lesions like pleural effusion or hydrocephalus.

> Diagnosis of paradoxical reaction should be made after careful exclusion of drug resistance and drug reaction.

\section{Author contributions}

Both authors jointly managed the patient. Initial writing of the case report was done by $\mathrm{AB}$ and the discussion part along with editing was done by SM.

\section{Conflict of interests}

The authors declare no conflicts of interest.

\section{Acknowledgements}

Written consent was obtained from the patient to publish the clinical details and images in this article.

\section{References}

1 Chien JW, Johnson JL. Paradoxical reactions in HIV and pulmonary TB. Chest 1998;114:933-6.

2 Chahed H, Hachicha H, Berriche A et al. Paradoxical reaction associated with cervical lymph node tuberculosis: predictive factors and therapeutic management. Int J Infect Dis 2017;54:4-7.

3 Garg RK, Malhotra HS, Kumar N. Paradoxical reaction in HIV negative tuberculous meningitis. J Neurol Sci 2014;340:26-36.

4 Cheng VC, Ho PL, Lee RA et al. Clinical spectrum of paradoxical deterioration during antituberculosis therapy in non-HIV-infected patients. Eur J Clin Microbiol Infect Dis 2002;21:803-9.

5 Cheng VCC, Yam WC, Woo PCY et al. Risk factors for development of paradoxical response during antituberculosis therapy in HIVnegative patients. Eur J Clin Microbiol Infect Dis 2003;22:597-602.

6 Cheng VCC. Paradoxical response during anti-tuberculosis therapy. Hong Kong Med J 2006;11:20-21.

Address for correspondence: Dr Ambarish Bhattacharya, 3A Sura East Road, Sadhan Apartment, Kolkata 700010, India. Email: ambarish2007dr@gmail.com 